\title{
Xenobióticos contaminantes presentes en el agua del refugio de vida silvestre pantanos de villa (distrito de chorrillos) y en el humedal de ventanilla (distrito de ventanilla) y su efecto sobre el crecimiento radicular y la composición cromosómica del ajo (allium sativum) y de la cebolla (allium cepa) utilizados como bioindicadores
}

\author{
Xenobiotic contaminants present in the water of the villa swamps of villa wildlife \\ refuge (chorrillos district) and in the humedal of ventanilla (ventanilla district) and \\ its effect on root growth and chromosome composition of garlic (allium sativum) \\ and onion (allium cepa) used as bioindicators
}

\section{Carlos Scotto ${ }^{1,3}$, Roxana Espino², Raúl Rondón ${ }^{3}$, César Arriola ${ }^{4}$ \& Mauro Quiñones ${ }^{1}$}

\section{Resumen}

Se determinó el efecto de la presencia de xenobióticos en muestras de agua de los humedales de los Pantanos de Villa en el distrito de Chorrillos (HVi) y de Ventanilla en el Distrito de Ventanilla (HVe) en el Departamento de Lima. En todas las muestras analizadas se disminuyó el crecimiento radicular comparado con los controles. Asimismo, hubo alteración de los índices de las fases mitóticas de los meristemos radiculares de cebolla (Allium cepa) y del ajo (Allium sativum). Se hizo germinar bulbos de cebolla y de ajo de cuatro sitios diferentes georreferenciados dentro de los humedales por un periodo de siete días versus un control negativo de agua sola desgasificada midiéndose el crecimiento de diez raíces promedio por bulbo germinado. Los porcentajes de inhibición del crecimiento radicular de la cebolla del HVi fueron M1 (80.2\%), M2 (55.6\%), M3 (63.1\%) y M4 (0\%). Y para el HVe M5 (39\%), M6 (30.9\%), M7 (68.9\%) y M8 (67\%). Y para el ajo para el HVe fueron M5 (56.9\%), M6 (9.4\%), M7 (59.2\%) y M8 (65.3\%). Asimismo, a las 72 horas se procesaron los ápices de las raicillas mediante la técnica de aplastamiento o "squash" y coloreadas con Orceína Acética Clorhídrico al 1\%. Las fases mitóticas fueron examinadas a 100X y 400X. Los resultados muestran una modificación de los índices mitóticos para ambos humedales. Pues no se pudo obtener ninguna división del ajo. Y para la cebolla en los $\mathrm{HVi}$ todos los puntos tuvieron menores valores al control (4.47). Y también variaron en los índices de las fases mitóticas: Pro=33.33, Met=33.33, Ana=11.11 e Tel=22.22. Los índices mitóticos para los puntos del HVe fueron inferiores en el punto M5 y superior en el punto M6 con respecto al control (4.25). Y también variaron en los índices de las fases mitóticas: Pro=25; Met=50; Ana=6.25 y Tel=18.75. Estos resultados demuestran que las aguas de ambos humedales contienen xenobióticos principalmente nitrato que afectarían el progreso normal del ciclo celular en el género Allium afectando el crecimiento radicular.

Palabras claves: Allium, xenobiótico, bioindicador, humedal, crecimiento radicular, citogenética.

\section{http://dx.doi.org/10.21503/cyd.v23i1.1862}

\footnotetext{
1Docente de la Facultad de Ciencias de la Universidad Ricardo Palma, Surco, Lima, carlos.scotto@urp.edu.pe

2Ingeniera Ambiental. Facultad de Ingeniería Ambiental de la Universidad Privada del Norte. Los Olivos, Lima, roxana_espino_1201@hotmail.com

3Químico. Docente de la Facultad de Ciencias Naturales y Matemática. Universidad Nacional Federico, El Agustino, Lima, rarondon@unfv.edu.pe 4Dr. Docente de la Universidad César Vallejo. Filial Este, Lima, carriolap15@gmail.com
} 


\section{Abstract}

The effect of the presence of xenobiotics in water samples from the wetlands of the Pantanos de Villa in the district of Chorrillos (HVi) and Ventanilla in the District of Ventanilla $(\mathrm{HVe})$ in the Department of Lima was determined. In all the samples analyzed, root growth was reduced compared to controls. Likewise, there were alterations in the indices of the mitotic phases of the root meristems of onion (Allium cepa) and garlic (Allium sativum). Onion and garlic bulbs were germinated from four different georeferenced sites within the wetlands for a period of seven days versus a negative control of single degassed water by measuring the growth of ten average roots per germinated bulb. The percentages of $\mathrm{HVi}$ onion root growth inhibition were M1 (80.2\%), M2 (55.6\%), M3 (63.1\%) and M4 (0\%). And for the HVe M5 (39\%), M6 (30.9\%), M7 (68.9\%) and M8 (67\%). And for garlic for HVe they were M5 (56.9\%), M6 (9.4\%), M7 (59.2\%) and M8 (65.3\%). Also, at 72 hours the apexes of the rootlets were processed by means of the squashing technique or "squash" and colored with $1 \%$ Hydrochloric Acetic Orcein. The mitotic phases were examined at 100X and 400X. The results show a modification of the mitotic indices for both wetlands. Well, no garlic division could be obtained. And for the onion in the $\mathrm{HVi}$, all the points had lower control values (4.47). And they also varied in the indices of the mitotic phases: Pro $=33.33$, Met $=$ 33.33, Ana $=11.11$ and Tel $=22.22$. The mitotic indices for the HVe points were lower at the M5 point and higher at the M6 point with respect to the control (4.25). And they also varied in the indices of the mitotic phases: Pro $=25$; Met $=50$; Ana $=6.25$ and Tel $=18.75$. These results demonstrate that the waters of both wetlands contain xenobiotics mainly nitrate that would affect the normal progress of the cell cycle in the Allium genus affecting root growth.

Keywords: Allium, xenobiotic, bioindicator, wetland, root growth, cytogenetics.

\section{INTRODUCCIÓN}

Los humedales son zonas donde el agua es el principal factor controlador del medio y la vida vegetal y animal asociada a él. Los humedales se dan donde la capa freática se halla en la superficie terrestre o cerca de ella o donde la tierra está cubierta por aguas. Las extensiones de marismas, pantanos y turberas, o superficies cubiertas de aguas, sean éstas de régimen natural o artificial, permanentes o temporales, estancadas o corrientes, dulces, salobres o saladas, incluidas las extensiones de agua marina cuya profundidad en marea baja no exceda de seis metros (1). Estos ecosistemas son considerados entre los más importantes del mundo por las funciones medio ambientales que realizan, como 
controlar los cursos de las corrientes de agua, participar en la regulación del carbono global, proporcionar hábitat a centenares de especies animales y vegetales, y poseer un importante valor cultural y recreacional (2).

El Refugio de Vida Silvestre Pantanos de Villa es un área natural protegida que se encuentra en el litoral del distrito de Chorrillos en el departamento de Lima (3). Es una reserva natural, que permite la anidación y el tránsito de aves migratorias y residentes. Es un sitio natural con abundancia en flora y fauna, perteneciente al Sistema Nacional de Áreas Naturales Protegidas del Estado-SINANPE, a cargo desde el 2008 del Servicio Nacional de Áreas Naturales Protegidas - SERNANP, organismo público descentralizado del Ministerio del Ambiente, parcialmente administrado por iniciativa propia por PROHVILLA, organismo de la Municipalidad Provincial de Lima (4). Presenta una altitud de 0 a 5 metros y una extensión actual de $276 \mathrm{Ha}$, encontrándose rodeada por un área de influencia conformada por asentamientos humanos, urbanizaciones, fábricas, clubes privados, establos, un camal y una avícola (5). El agua de los Pantanos de Villa es del tipo salobre, con una salinidad variable, siendo mayor próximo al litoral marino; la concentración de sales registrada es de 11 a 60 ppm. El color del agua varía desde pardo claro hasta oscuro, con una gran abundancia de materia orgánica en descomposición. El pH fluctúa entre 6 y 8.5. La contaminación de las aguas se debe principalmente a la presencia de aguas servidas, transportadas a través de canales laterales y que van a dar a los cuerpos de agua mayores (6) (Figura 1). 


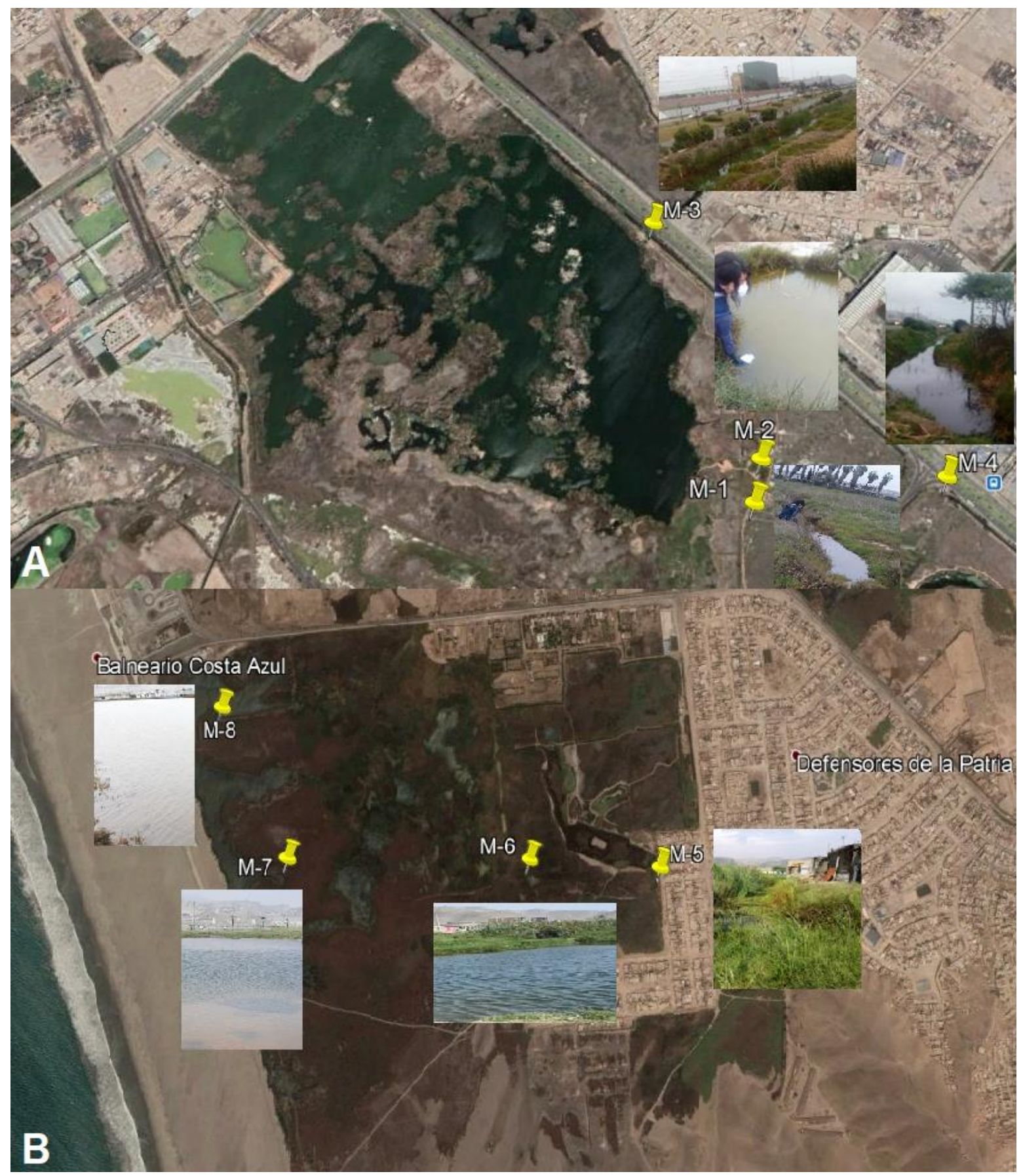

Figura 1. Puntos de monitoreo. A. Refugio de vida silvestre Pantanos de Villa: M1(Lat.12.20998197/Long.-76.99027356). M2(Lat.-12.20937107/Long.-76.99012991. M3(Lat.12.20576113/Long.-76.99145749). M4(Lat.-12.20973621/Long.-76.98756031). B. Humedal de Ventanilla: M5(Lat.-11.878530/Long.-77.141242). M6(Lat.-11.878304/Long.77.127636). M7 (Lat.-11.877745/Long.-77.151865). M8 (Lat.-11.874239/Long.-77.153399).

Los Humedales de Ventanilla se encuentran ubicados en la parte centro occidental del distrito de Ventanilla, provincia constitucional del Callao en el departamento de Lima. Actualmente abarca un área de $538,68 \mathrm{Ha}$ de las $653,22 \mathrm{Ha}$ con que contaba, a 
consecuencia de la ocupación por parte de asentamientos humanos y la ampliación del área agrícola. Limitan por el oeste con la playa de Ventanilla, la cual es de tipo arenoso, con un ancho aproximado de $300 \mathrm{~m}$ (7). Está conformada por un conjunto de sistemas lagunares permanentes y temporales que se alimentan en condiciones naturales por filtraciones del río Chillón y del litoral marino. Constituye refugios, sitios de reproducción y alimentación especialmente de aves acuáticas endémicas y migratorias. A pesar de esto, los cuerpos de agua presentan una problemática por las invasiones y sobrepoblación en zona cercanas a la misma (8) (Figura 1).

\section{Xenobiótico}

Un xenobiótico es una estructura química que no existe en la naturaleza, sino que ha sido desarrollada por la actividad del hombre en un laboratorio, los cuales han aparecido en el medio ambiente en los últimos 200 años (9). En 1947, Charlotte Auerbach público que los xenobióticos producen alteraciones en los cromosomas (10). Son principalmente introducidos por actividades humanas y su uso se ha incrementado en las últimas décadas de forma considerable $Y$ que finalmente van al ambiente, ya sea intencionadamente o por accidente (11).

\section{Bioindicador}

El uso de bioindicadores se está proponiendo como una nueva herramienta para conocer la calidad del agua. Por ende, para obtener una evaluación integral será necesario realizar conjuntamente análisis fisicoquímicos o pruebas de toxicidad (12,13 y 14).

\section{Crecimiento radicular en el género Allium}

Actualmente diversos ensayos ecotoxicológicos utilizan a los bulbos de $A$. cepa y $A$. sativum. Cuando un bulbo de Allium se rehidrata, se estimula el crecimiento de las células y se elongan las raíces de la planta, pero si la hidratación se realiza en presencia de sustancias toxicas este crecimiento puede inhibirse. La cuantificación de este efecto puede realizarse mediante la evaluación de la inhibición del crecimiento promedio de raíces respecto a la longitud promedio de las raíces del control sin contaminantes (15) (Figura 2). Castillo Morales (16) menciona que para obtener el porcentaje de efecto de inhibición sobre el crecimiento radicular se debe realizar la siguiente Fórmula: 

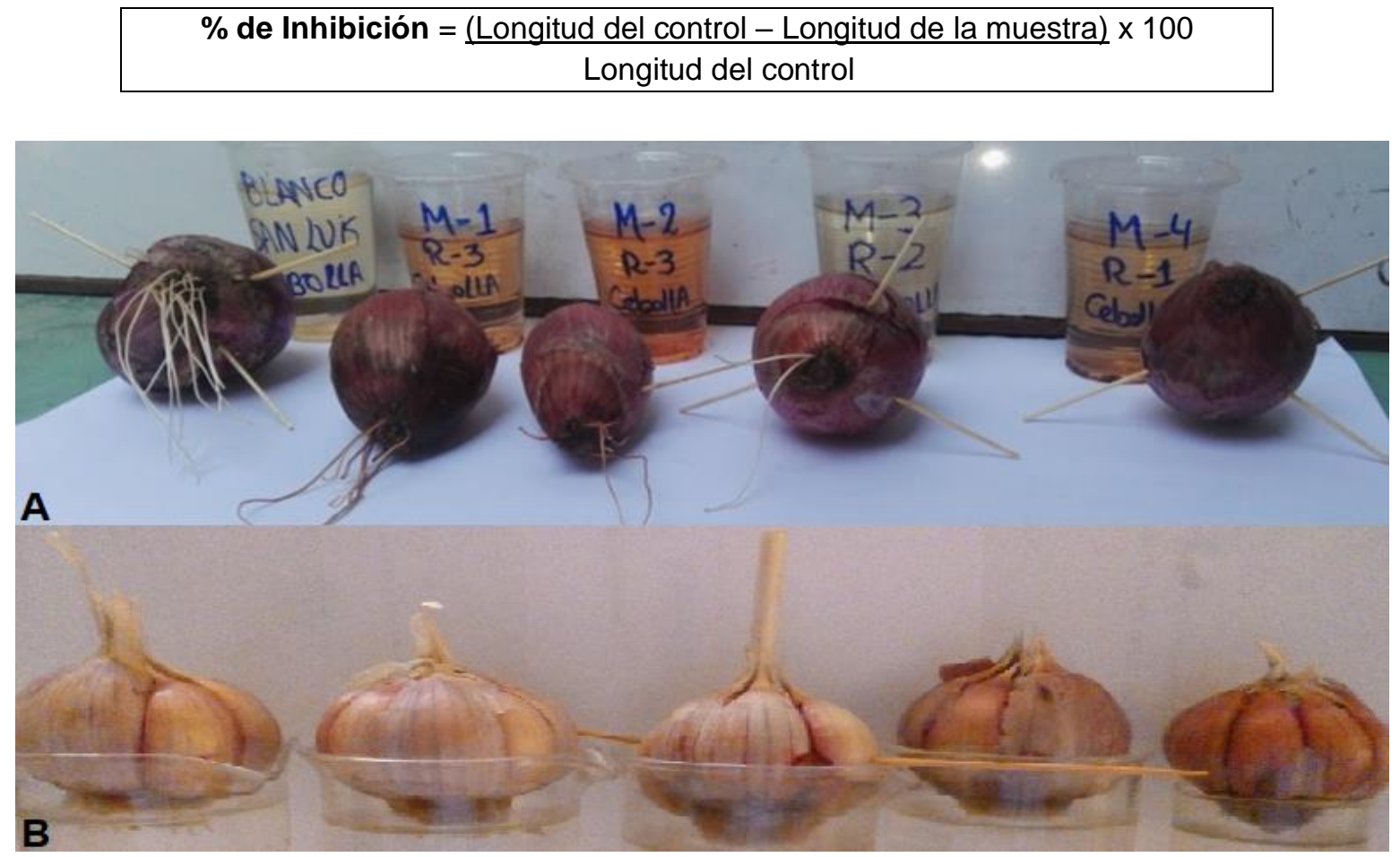

Figura 2. Muestras de bulbos para su enraizamiento en las cuatro muestras de agua por humedal y un control: A. Cebolla (Allium cepa). B. Ajo (Allium sativum) (Fotografía de propia autoría).

\section{Número cromosómico en el género Allium}

Tanto la cebolla (Allium cepa) como el ajo (Allium sativum) su número de cromosómico es de 16. Con la mitosis comienza un proceso de compactación del ADN que alcanza su máximo nivel en la metafase al visualizarse los cromosomas. Los cromosomas se tiñen fácilmente cuando están condensados y pueden ser individualizados con el microscopio óptico. Este ensayo se denomina "Allium Test" (17 y 18) (Figura 3).

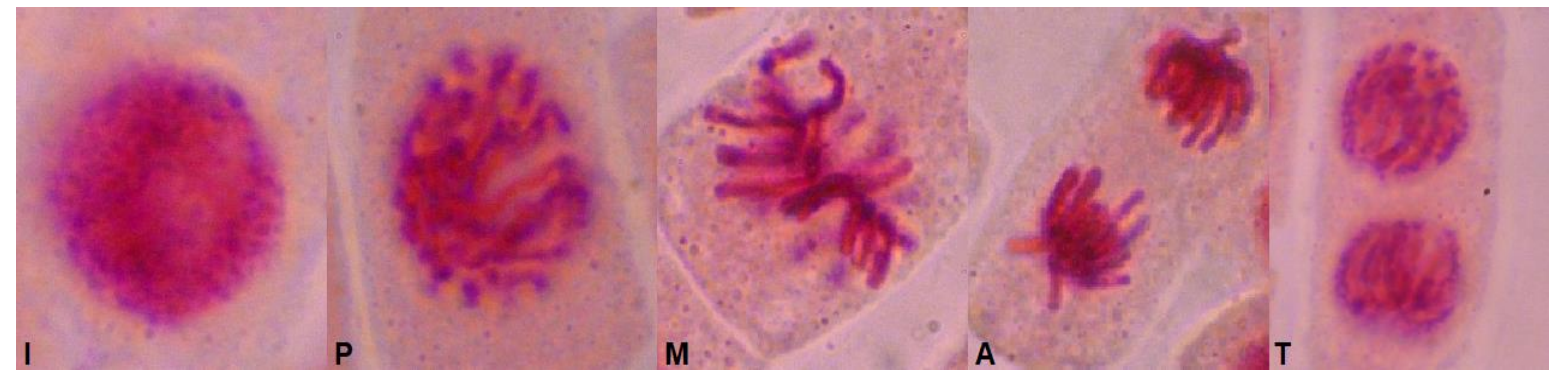

Figura 3. Fases de la mitosis en cebolla (Allium cepa): Interfase (I), Profase (P), Metafase (M), Anafase (A) y Telofase (T) (400X) (Fotografía de propia autoría). 


\section{MATERIALES Y MÉTODOS}

\section{Análisis de la calidad fisicoquímica del agua de los humedales}

Los puntos de monitoreo se realizaron entre los meses de enero y febrero del año 2018 según el Protocolo Nacional para el Monitoreo de la Calidad se los Recursos Hídricos Superficiales donde se procedió a la toma de muestras usando la indumentaria adecuada como botas de jebe, guantes de nitrilo y mascarilla. Además de vestir polo manga larga y un chaleco protector.

Se tomó una muestra de agua por cada punto de monitoreo para luego realizar su análisis de los siguientes parámetros físicoquímicos: Cloruros (mg/L), Amonio (mg/L), Calcio (mg/L), Nitratos (mg/L), Oxígeno Disuelto (mg/L), Sólidos Totales Disueltos (mg/L), Conductividad $(\mu \mathrm{s} / \mathrm{cm})$, Turbidez (NTU) y pH (Tabla 1). Para el recojo de las muestras se usaron botellas de poliestireno estériles, que fueron transportados en un "cooler". Antes de la toma de la muestra del agua se etiquetó y rotuló el envase. Los envases fueron previamente enjuagados tres veces como mínimo con el agua del humedal y después se sumergió el frasco a $20 \mathrm{~cm}$ de profundidad colocando la boquilla contra la corriente y se esperó por un lapso de tiempo hasta que esta se llenó. Finalmente, se cerró el envase y se le colocó en el cooler para su transporte y procesamiento en el laboratorio.

\section{Medición del crecimiento radicular de los bulbos de cebolla (Allium cepa) y el ajo (Allium sativum)}

Se emplearon un total de tres bulbos de ajos y tres bulbos de cebollas (Triplicado) por muestra de agua colectada. Asimismo, se realizó un ensayo por triplicado para el control con agua San Luis sin gas. Se utilizó bulbos que fueran del mismo tamaño en lo posible y que no tengan raíces para que se facilite el proceso del análisis del crecimiento radicular. Estos dos bioindicadores fueron distribuidos para su germinación en 100ml de agua colectada. Se utilizó un control negativo con agua San Luis® ${ }^{\circledR}$ in gas (Figura 4).

Se realizó un registro durante las 24, 48, 96, 120, 144 y 168 horas del crecimiento radicular de cada ajo y cebolla por muestra. Y se midió diez raíces por bulbo germinado. Las medidas fueron medidas en milímetros con un vernier o "Pie de rey" (Figuras 5 y 6). 


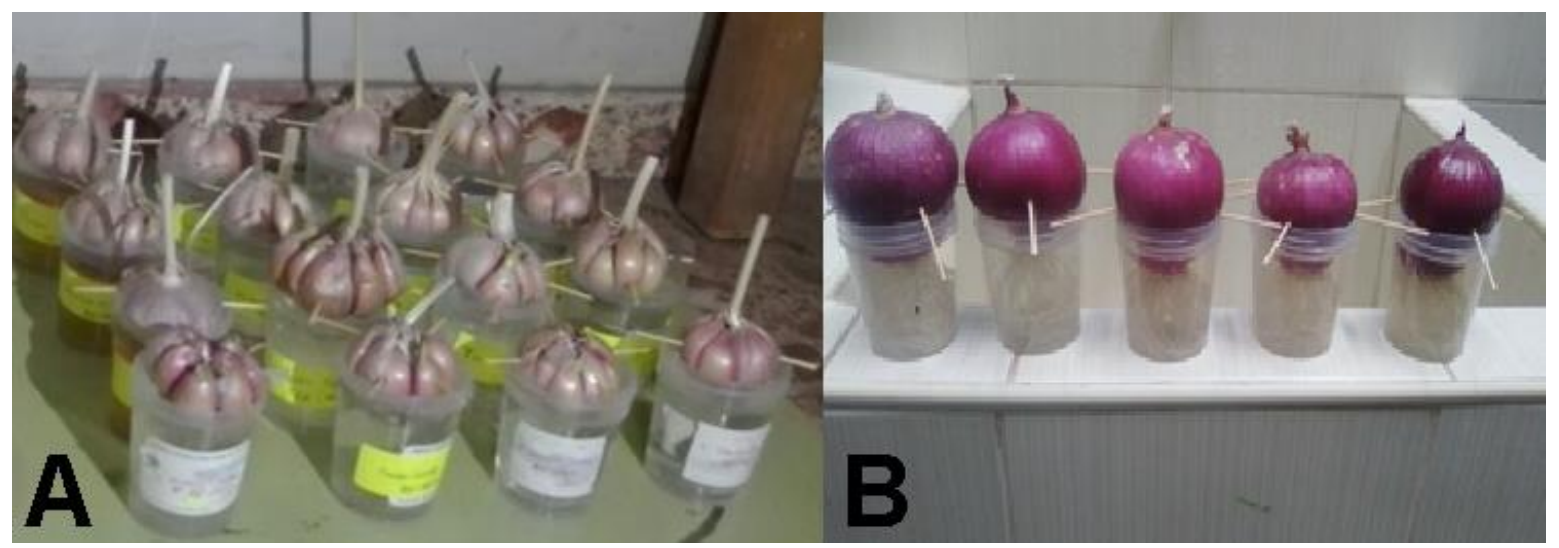

Figura 4. Muestras de bulbos para su enraizamiento: A. Ajo (Allium sativum). B. Cebolla (Allium cepa).

\section{Análisis citogenético}

Para el análisis citogenético se escogió las raíces de ajo y de cebolla a las 72 horas de germinación y a una temperatura de ambiente de 18 a $20^{\circ} \mathrm{C}$ promedio. Se cortó $0.5 \mathrm{~cm}$ de los ápices de las raíces seleccionadas y se las puso en una placa Petri. Se añadió aproximadamente 20 gotas de fijador Carnoy por 3 horas. Se las colocó luego en una luna de reloj y se le cubrió con cinco gotas de la solución de Orceína Acética y dos gotas de Ácido clorhídrico $1 \mathrm{~N}$. Se tomó con una pinza de madera y se la flameó en el mechero hasta hervir por tres veces y se le dejó enfriar 5 minutos. Luego se colocó los ápices encima de una laminilla portaobjeto y se cubrió con una lámina cubreobjeto. Y se presionó con fuerza el dedo pulgar "squash" presionando contra la laminilla y se absorbió el residuo del colorante de los bordes con un papel toalla. Se colocó la preparación en el microscopio y se buscó la zona en que estén las células del meristemo a 100X de aumento. Una vez localizada alguna fase de la mitosis se pasó a un aumento de 400X para una observación más detallada. Y se realizó las tomas fotográficas de los cariotipos empleando un teléfono móvil smarphone con una resolución de 12 megapíxeles Marca LG (Figura 3). Se realizó el conteo del número de células que se encuentran en interfase $(I)$, Profase $(P)$, Metafase $(M)$, Anafase $(A)$ y Telofase $(\mathrm{T})$ y poder hallar el porcentaje de índice mitótico de cada fase (Tabla 2).

\section{Análisis estadístico}

Se utilizó el programa de computación Excel (Versión 1911) para el procesamiento de los datos y generación de gráficos. 


\section{RESULTADOS}

En la Tabla 1, se determinó parámetro fisicoquímico como fueron: Temperatura $\left({ }^{\circ} \mathrm{C}\right), \mathrm{pH}$, Conductividad $(\mu \mathrm{S} / \mathrm{cm})$, Solidos suspendidos totales $(\mathrm{mg} / \mathrm{L})$, Salinidad $(\mathrm{g} / \mathrm{kg})$, Oxígeno Disuelto ( $\left.\mathrm{mg} \mathrm{O}_{2} / \mathrm{L}\right)$, Turbidez (NTU), Cloruros (mg/L), Dureza Cálcica $\left(\mathrm{mg} \mathrm{CaCO}_{3} / \mathrm{L}\right)$, Nitratos $\left(\mathrm{mg} / \mathrm{L} \mathrm{NO}_{3}\right)$ y Amonio $\left(\mathrm{mg} / \mathrm{L} \mathrm{NH}_{3}\right)$.

Todos los datos fueron comparados con el ECA de agua Categoría 4 y subcategoría E1 para humedales (19).

TABLA 1. Parámetros fisicoquímicos analizados por punto de muestreo.

\begin{tabular}{|c|c|c|c|c|c|c|c|c|c|c|}
\hline \multirow[b]{2}{*}{$\begin{array}{c}\text { Parámetro } \\
\text { fisicoquímico }\end{array}$} & \multicolumn{4}{|c|}{ PANTANOS DE VILLA } & \multicolumn{4}{|c|}{ HUMEDAL DE VENTANILLA } & \multirow[b]{2}{*}{ Control } & \multirow{2}{*}{$\begin{array}{c}\text { ECA } \\
\text { (Cat.4) } \\
\begin{array}{c}\text { (Subcat. } \\
\text { E1) }\end{array}\end{array}$} \\
\hline & M1 & M2 & M3 & M4 & M5 & M6 & M7 & M8 & & \\
\hline $\begin{array}{c}\text { Temperatura } \\
\left({ }^{\circ} \mathrm{C}\right)\end{array}$ & 26.40 & 26.10 & 26.30 & 26.40 & 27.80 & 28.50 & 29.40 & 29.20 & 26.50 & $\Delta 3$ \\
\hline $\mathrm{pH}$ & 8.09 & 7.98 & 7.75 & 7.70 & 7.17 & 7.54 & 8.86 & 8.91 & 7.66 & $6.5-9.0$ \\
\hline $\begin{array}{c}\text { Conductividad } \\
(\mu \mathrm{S} / \mathrm{cm})\end{array}$ & 3750.67 & 3292.33 & 3873.67 & 3866 & 8530 & 1438 & 25600 & 49200 & 84.20 & 1000 \\
\hline $\begin{array}{c}\text { Sólidos } \\
\text { suspendidos } \\
\text { totales (mg/L) }\end{array}$ & $19.3 \times 10^{-6}$ & $17.2 \times 10^{-6}$ & $19.4 \times 10^{-6}$ & $19.4 \times 10^{-6}$ & $42.6 \times 10^{-6}$ & $71.9 \times 10^{-6}$ & $128 \times 10^{-6}$ & $246 \times 10^{-6}$ & $0.4 \times 10^{-6}$ & $\leq 25$ \\
\hline Salinidad(g/kg) & 4.86 & 2.44 & 7.50 & 7.12 & $4.71 \times 10^{-9}$ & $15.39 \times 10^{-9}$ & $23.18 \times 10^{-9}$ & $63.09 \times 10^{-9}$ & 0.02 & \\
\hline $\begin{array}{c}\text { Oxígeno } \\
\text { Disuelto } \\
\left.\text { (mg O } \mathrm{m}_{2} / \mathrm{L}\right)\end{array}$ & 8.19 & 8.16 & 7.99 & 7.89 & 7.39 & 7.60 & 7.54 & 7.27 & 4.29 & $\geq 4$ \\
\hline Turbidez (NTU) & 2.68 & 4.09 & 30.40 & 29.80 & 14.30 & 5.36 & 13.30 & 25.30 & 0.11 & \\
\hline $\begin{array}{c}\text { Cloruros } \\
\text { (mg/L) }\end{array}$ & 2694 & 1355.50 & 4152.50 & 3941 & 2610 & 8518 & 12833.04 & 34923.04 & 11.48 & \\
\hline $\begin{array}{c}\text { Dureza Cálcica } \\
(\mathrm{mg} \mathrm{CaCO} / \mathrm{L})\end{array}$ & 124.65 & 68.35 & 178.05 & 170.20 & 0.03 & 0.26 & 0.162 & 0.18 & 0.50 & \\
\hline $\begin{array}{c}\text { Nitratos } \\
\left.(\mathrm{mg} / \mathrm{L} \mathrm{NO})_{3}\right)\end{array}$ & 150.20 & 124.35 & 34.38 & 162.70 & 9.36 & 12.36 & 86.09 & 14.04 & 1.64 & 13 \\
\hline $\begin{array}{c}\text { Amoniaco } \\
\text { Total } \\
(\mathrm{mg} / \mathrm{L} \mathrm{NH})_{3}\end{array}$ & 0.055 & 0.166 & 0.315 & 0.208 & 0.607 & 2.010 & 0.983 & 3.523 & 0.074 & \\
\hline
\end{tabular}

$(\mathrm{M})=$ Punto de muestreo

$\left({ }^{*}\right)=$ ppt,

$\Delta 3=$ Variación de $3^{\circ} \mathrm{C}$ respecto al promedio mensual multianual del área evaluada en los últimos 05 años como máximo y de 01 año como mínimo.

En la Figura 5, se muestra el crecimiento radicular de la Cebolla (Allium cepa) para las muestras de agua del Refugio de vida silvestre Pantanos de Villa: M1, M2, M3 y M4 (Sin crecimiento radicular) y del Humedal de Ventanilla: M5, M6, M7, M8 y del Control (Agua desgasificada). 
En la Figura 6, sólo se muestra el crecimiento radicular ajo (Allium sativum) para las muestras de agua del Humedal de Ventanilla: M5, M6, M7, M8 y el Control. No hubo germinación del ajo para ninguna de las muestras de Refugio de vida silvestre Pantanos de Villa.

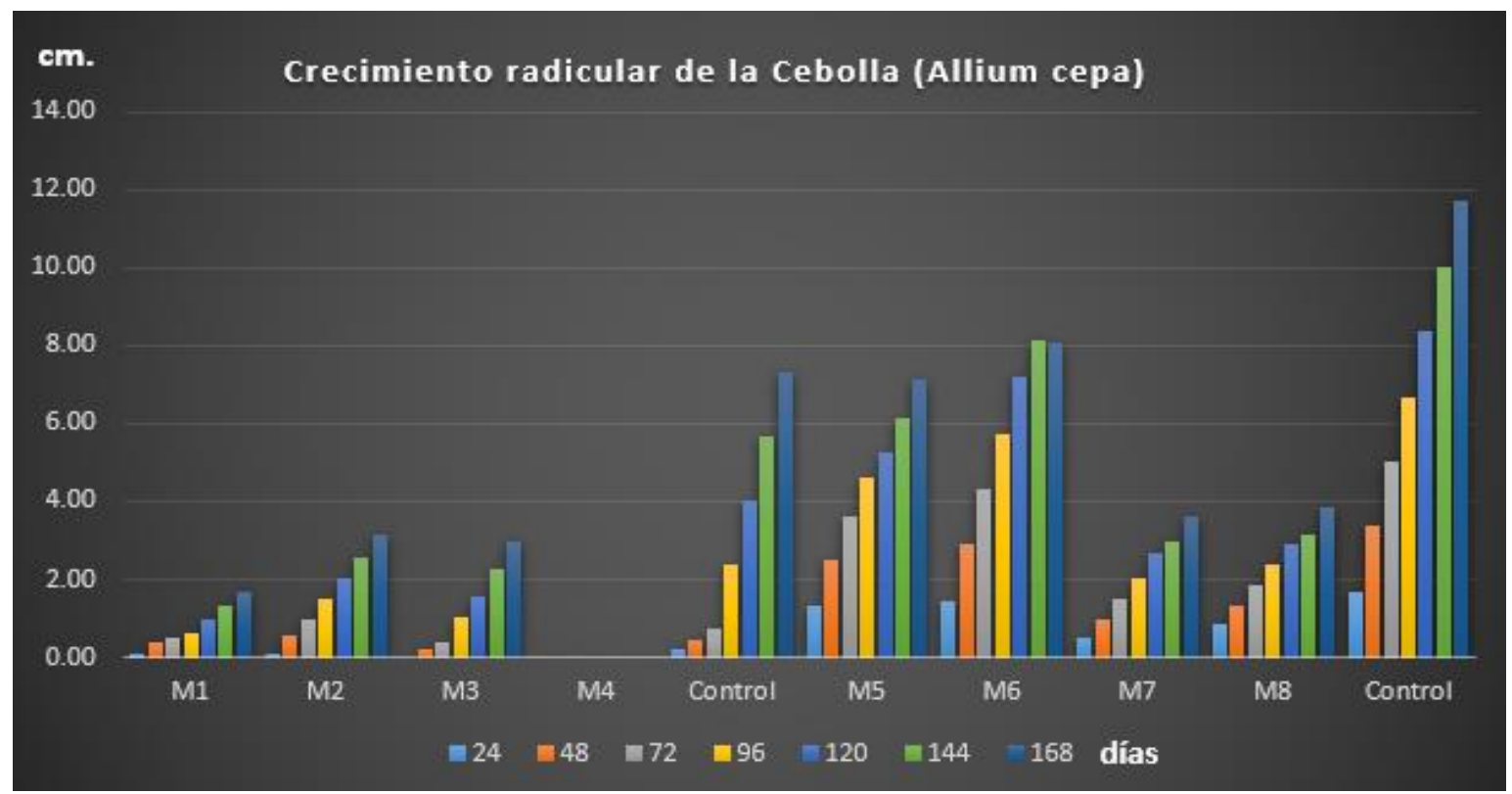

FIgURA 5. Crecimiento radicular de la Cebolla (Allium cepa). Puntos de monitoreo del Refugio de vida silvestre Pantanos de Villa: M1, M2, M3 y M4 (Sin crecimiento radicular). Puntos de monitoreo del Humedal de Ventanilla: M5, M6, M7, M8 y un Control (Agua desgasificada).

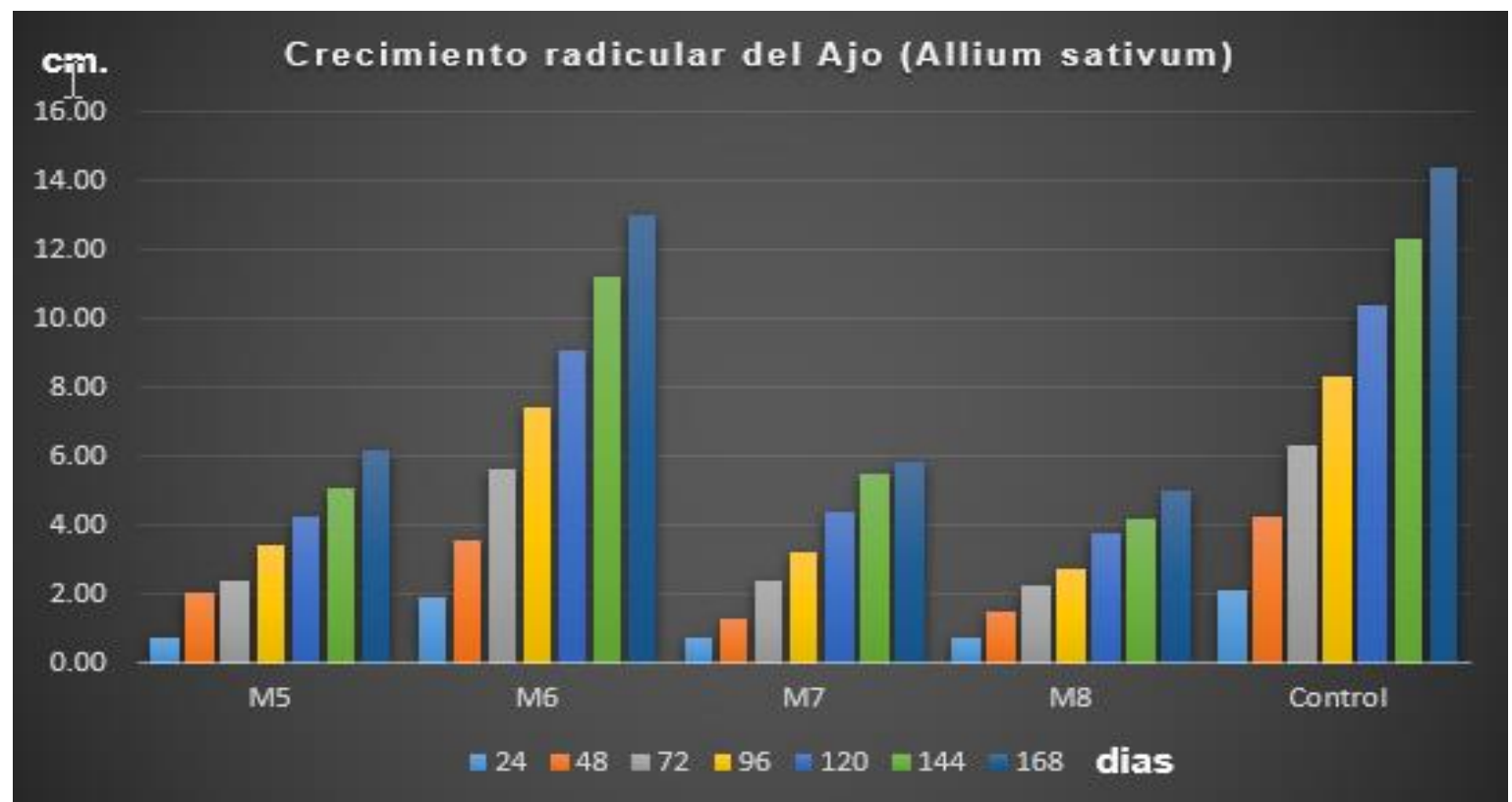

FIGURA 6. Crecimiento radicular del ajo (Allium sativum). Puntos de monitoreo del Humedal de Ventanilla: M5, M6, M7, M8 y un Control (Agua desgasificada). 
En la Tabla º2, se muestra el número de los tipos células mitóticas (Interfase, Profase, Metafase, Anafase y telofase) obtenidas de las muestras los puntos de monitoreo del Refugio de vida silvestre Pantanos de Villa (M1, M2 y M3) y de los puntos de monitoreo del Humedal de Ventanilla (M5 y M6) versus un Control (Agua desgasificada). Todo se realizó por duplicado.

TABLA 2. Índices mitóticos con repetición obtenidos por punto de muestreo con Cebolla.

\begin{tabular}{|c|c|c|c|c|c|c|c|c|c|c|c|c|}
\hline $\begin{array}{l}\text { PANTANOS } \\
\text { DE VILLA }\end{array}$ & I & $\mathbf{P}$ & M & A & $\mathbf{T}$ & B & C & $(C / B)$ & $(P / C)$ & $(\mathrm{M} / \mathrm{C})$ & $(A / C)$ & $(\mathrm{T} / \mathrm{A})$ \\
\hline M1 & 198 & 1 & 1 & 2 & 1 & 203 & 5 & 2.46 & 20 & 20 & 40 & 20 \\
\hline M1 & 237 & 3 & 1 & 1 & 1 & 243 & 6 & 2.47 & 50 & 16.667 & 16.67 & 16.67 \\
\hline M2 & 183 & 0 & 3 & 0 & 2 & 188 & 5 & 2.66 & 0 & 60 & 0 & 40 \\
\hline M2 & 204 & 2 & 1 & 1 & 1 & 209 & 5 & 2.39 & 40 & 20 & 20 & 20 \\
\hline M3 & 170 & 2 & 3 & 2 & 1 & 178 & 8 & 4.49 & 25 & 37.5 & 25 & 12.5 \\
\hline M3 & 168 & 1 & 3 & 3 & 1 & 176 & 8 & 4.55 & 12.50 & 37.50 & 37.50 & 12.50 \\
\hline Control & 385 & 6 & 6 & 2 & 4 & 403 & 18 & 4.47 & 33.33 & 33.33 & 11.11 & 22.22 \\
\hline $\begin{array}{l}\text { HUMEDAL DE } \\
\text { VENTANILLA }\end{array}$ & I & $\mathbf{P}$ & $\mathbf{M}$ & A & $\mathbf{T}$ & B & C & $(C / B)$ & $(P / C)$ & $(\mathrm{M} / \mathrm{C})$ & $(\mathrm{A} / \mathrm{C})$ & (T/A) \\
\hline M5 & 200 & 2 & 1 & 1 & 1 & 205 & 5 & 2.44 & 40 & 20 & 20 & 20 \\
\hline M5 & 171 & 2 & 1 & 0 & 2 & 176 & 5 & 2.84 & 40 & 20 & 0 & 40 \\
\hline M6 & 136 & 2 & 14 & 1 & 2 & 155 & 19 & 12.26 & 10.53 & 73.68 & 5.26 & 10.53 \\
\hline M6 & 165 & 2 & 16 & 2 & 1 & 186 & 21 & 11.29 & 9.52 & 76.19 & 9.52 & 4.76 \\
\hline Control & 338 & 4 & 8 & 1 & 3 & 354 & 16 & 4.52 & 25.00 & 50.00 & 6.25 & 18.75 \\
\hline $\begin{array}{l}\text { Número de célt } \\
\text { B=Número de } \\
\text { M/C=Índice meta } \\
\text { Refugio de vida } \\
\text { Ventanilla: M5 y }\end{array}$ & las $p$ & & antro & 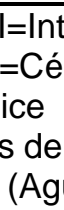 & & & & & $M=$ Meta & $e, \quad A=A$ & ase, $\mathrm{T}$ & Telofase \\
\hline
\end{tabular}

\section{DISCUSION}

Todos los resultados obtenidos de los análisis de los principales parámetros fisicoquímicos de importancia biológica de los puntos de monitoreo de ambos humedales (M1 a M8) fueron comparados con el ECA del agua (19). Para el caso de la conductividad, la norma indica que no debe sobrepasar el valor de 1000us/cm. Sin embargo, en la Tabla 1 los resultados obtenidos en promedio de las ocho muestras de agua analizadas de ambos humedales fueron en promedio casi de 0.5 (M6) a 5 (M8) uS/cm veces más alto con respecto a la norma (Control = $84.2 \mathrm{uS} / \mathrm{cm})$. Asimismo, el pH tuvo valores ligeramente alcalinos desde $7.17 \mathrm{a}$ 8.86 estando dentro del rango de la norma. Por otro lado, el oxígeno disuelto estuvo dentro del rango aceptable con valores entre 7.27 a $8.19 \mathrm{mgO}_{2} / \mathrm{L}$. Valores superiores al ECA agua ( $\geq 4 \mathrm{mgO}_{2} / \mathrm{L}$ ). En cuanto a los sólidos totales suspendidos los valores fueron bastante 
ínfimos desde $17.2 \times 10^{-6}$ hasta $246 \times 10^{-6} \mathrm{mg} / \mathrm{L}$ comparada con el ECA $(\leq 25)$. Y el rango de turbidez estuvo entre 4.09 a 30.4 NTU (No existe dato de ECA). Al ser verano, las temperaturas registradas estuvieron en el rango de 26.1 a $29.4^{\circ} \mathrm{C}$ (Tabla 1 ).

En cuanto a la presencia de Nitratos, solamente los puntos M6 y M8 estuvieron cerca al valor del ECA agua que es de $13 \mathrm{mg} / \mathrm{L} \mathrm{NO}$. Y el punto $\mathrm{M} 5$ fue el más bajo (9.36 mg/L NO $\mathrm{NO}_{3}$. Los otros puntos de monitoreo (M1, M2, M3, M4, M5 y M7) tuvieron un rango entre 34.38 y 162.7. El valor del control fue de $1.64 \mathrm{mg} / \mathrm{L} \mathrm{NO}_{3}$. Esta presencia de exceso de nitrato en estos puntos de muestreo podría deberse solamente a dos causas: Presencia cercana de zonas de producción agrícola por la utilización de abonos nitrogenados y/o excrementos de animales de granja. $O$ por el vertimiento de aguas residuales a través de canales 0 desagües clandestinos.

Un dato divergente entre ambos humedales fue en cuanto a la salinidad. Por un lado, los cuatro puntos de monitoreo de los humedales de Ventanilla (M5 al M8) registraron valores muy bajos entre $4.7 \times 10^{-9}$ a $63 \times 10^{-9} \mathrm{~g} / \mathrm{Kg}$. Mientras que los cuatro puntos de monitoreo de los Pantanos de Villa (M1 al M4) registraron valores altos entre 2.44 a $7.5 \mathrm{~g} / \mathrm{Kg}$ comparado con el Control que fue de $0.02 \mathrm{~g} / \mathrm{Kg}$. Esto evidencia una mayor infiltración salobre en los Pantanos de Villa con respecto a los humedales de Ventanilla. Pudiendo explicar el por qué no se evidencio crecimiento radicular alguno en el ajo (Figura 6). Además, se obtuvieron las menores elongaciones de raíces en la cebolla -incluso el punto M4 no creció- con respecto al crecimiento radicular para el humedal de Ventanilla. Por último, ningún punto de monitoreo de ambos humedales superó en crecimiento radicular al control (Figura 5).

A pesar de no existir dato de ECA de agua por la presencia de cloruros para humedales. Esta fue muy alta en los puntos de monitoreo M7 y M8 con valores de 12833.04 y 34923.04 $\mathrm{mg} / \mathrm{L}$ respectivamente. Siendo los más bajos los puntos de monitoreo M1 y M5 con valores de 2694 y 2610 mg/L (Control = 11.48 mg/l). En cuanto al carbonato de calcio se evidenció una dicotomía notoria. Por un lado, los cuatro puntos de monitoreo de los humedales de Ventanilla (M5 al M8) registraron valores muy bajos con un rango entre 0.03 a $0.18 \mathrm{mg} / \mathrm{L}$. Mientras que los cuatro puntos de monitoreo de los Pantanos de Villa (M1 al M4) registraron valores altos entre 68.35 a $178.05 \mathrm{mg} / \mathrm{L}$ comparado con el Control que fue de $0.5 \mathrm{mg} / \mathrm{L}$ (Tabla 1).

Si bien el Decreto Supremo N004-2017-MINAM (20) no contempla valores del amoniaco total para humedales en el ECA del agua. Se buscó correlacionar la concentración de 
amoniaco total en función del $\mathrm{pH}$, la temperatura y la salinidad para la protección de la vida acuática. Es así que medido en $\mathrm{g} / \mathrm{Kg}$ los valores deberían ser: A una temperatura de $25^{\circ} \mathrm{C}$ : $\mathrm{pH} 7$ (3.37), pH 7.5(1.08), pH 8(0.354), pH 8.5(0.125) y pH 9 (0.053). A una temperatura de $30^{\circ} \mathrm{C}$ : pH 7(2.39), pH 7.5(0.767), pH 8(0.256), pH 8.5(0.094) y pH 9 (0.043). Los puntos M1, M2, M3 y M4 de los Pantanos de Villa estuvieron cerca a los $25^{\circ} \mathrm{C}$ y un $\mathrm{pH}$ de 8.0. Por lo tanto, todos sus valores superaron los $0.354 \mathrm{~g} / \mathrm{Kg}$ de amoniaco total. Los puntos M5, M6, M7 y M8 de los Humedales de Ventanilla estuvieron cerca a los $30^{\circ} \mathrm{C}$ y con un $\mathrm{pH}$ de 7.0 para M5, pH 7.5 para M6 y un pH 9.0 para los puntos M7 y M8. Sin embargo, todos sus valores fueron muy inferiores cuyos valores fueron $0.6,2.01,0.983$ y $0.074 \mathrm{~g} / \mathrm{Kg}$ de amoniaco total.

En cuanto al crecimiento radicular con cebolla los resultados muestran menores crecimientos radiculares para todos los puntos de monitoreo de los dos humedales comparado con los controles respectivos en las siete mediciones realizadas de 24, 48, 72 , 96, 144 y 168 horas. Los puntos de monitoreo de los Pantanos de Villa alcanzaron un máximo de crecimiento radicular promedio (Se hizo tres replicaciones) en centímetros de: M1 (1.7), M2 (3.16), M3 (2.98) y M4(0.0) comparado con el control que fue de $8.6 \mathrm{~cm}$. Cuyos valores fueron un 8 a 2.7 veces menor. El punto M4 no creció ninguna raíz medible. Los porcentajes de inhibición del crecimiento radicular fueron: M1 (80.2\%), M2 (55.6\%), M3 (63.1\%) y M4 (100\%). Para los puntos de monitoreo de los humedales de Ventanilla éstos alcanzaron un máximo crecimiento radicular promedio (Se hizo tres replicaciones) en centímetros de: M5 (7.13), M6 (8.08), M7 (3.64) y M8 (3.85) comparado con el control que fue de $11.69 \mathrm{~cm}$. Cuyos valores fueron un 3.2 a 1.5 veces menor. Los porcentajes de inhibición del crecimiento radicular fueron: M5 (39\%), M6 (30.9\%), M7 (68.9\%) y M8 (67\%).

Es notorio una mayor inhibición del crecimiento de la raíz de la cebolla en las aguas procedentes de los pantanos de Villa con respecto al humedal de Ventanilla (Figura 5).

Para el ajo, ninguno de los puntos de monitoreo de los Pantanos de Villa creció. Habiendo un xenobiótico contaminante que afecta seriamente la germinación de ésta liliácea. Para los puntos de monitoreo de los humedales de Ventanilla éstos alcanzaron un máximo crecimiento radicular promedio (Se hizo tres replicaciones) en centímetros de: M5 (6.18), M6 (13.01), M7 (5.85) y M8 (4.98) comparado con el control que fue de $14.35 \mathrm{~cm}$. Cuyos valores fueron un 2.9 a 1.1 veces menor. Los porcentajes de inhibición del crecimiento radicular fueron: M5 (56.9\%), M6 (9.4\%), M7 (59.2\%) y M8 (65.3\%) (Figura 6). 
No se pudo obtener ninguna división del ajo para ninguna de las ocho muestras de ambos humedales. Con cebolla, solamente las muestras M1, M2, M3, M5 y M6; se pudieron obtener células en división celular. Así los índices mitóticos para los puntos de los Pantanos de Villa fueron inferiores en los puntos M1 y M2 y ligeramente más alto en el punto M3 con respecto al control 4.47. Y de los índices de las fases tuvieron valores superiores e inferiores con respecto al control: IPro = 33.33; IMet = 33.33; IAna = 11.11 e ITel=22.22. Los índices mitóticos para los puntos del humedal de Ventanilla fueron inferiores en el punto M5. Y superior en el punto M6 con respecto al control 4.25. Y de los índices de las fases tuvieron valores superiores e inferiores con respecto al control: IPro = 25; IMet = 50; IAna = 6.25 e $\mathrm{IT}=18.75$ (Tabla 2$)$.

Finalmente, de la Figura 1 geográficamente para los pantanos de Villa el punto M1 está a 30 metros del ingreso al humedal. El punto M2 estaba cerca a los caminos de tránsito de los usuarios que visitan los humedales. El punto M3 se encuentra cerca de la pista y es el hábitat de peces y de aves. Y el punto M4 estaba a 20 metros del lugar donde los pobladores aledaños arrojan desperdicios. En los cuatro puntos sus conductividades fueron superiores a los $3200 \mathrm{uS} / \mathrm{cm}$ siendo valores que superan los $2 \mathrm{~g} / \mathrm{L}$ de contenido salino del agua. La fitoxicicidad se acentúa con la elevación de la temperatura. En este caso fue superior a los 26드. La salinización determina el incremento del potencial osmótico (estrés hídrico), con lo cual se dificulta la capacidad de absorción de agua por parte de las raíces (21 y 22). Asimismo, en los cuatro puntos muestreados existen altos contenido de Nitratos por encima del ECA. En cuanto al amoniaco total solamente los puntos M1, M2, M3 y M4 de los Pantanos de Villa $\left(25^{\circ} \mathrm{C}\right.$, $\mathrm{pH}$ de 8.0 aproximado) sus valores superaron los $0.354 \mathrm{~g} / \mathrm{Kg}$. Se sabe que el amoniaco se transforma en nitrito y este en nitrato. Podría de este modo, existir una contaminación difusa del ion nitrato (NO3-) hacia este humedal. Así probablemente, los mecanismos de llegada del contaminante nitrato serían a partir del suelo por la infiltración de las aguas superficiales contaminadas de acequias o desagües clandestinos que afectan a las aguas subterráneas que lo alimentan. También podría deberse al uso de fertilizantes de origen animal como el estiércol de corrales de animales aledaños al humedal y que llegan por canales o desagües. Para el humedal de Ventanilla, el punto M5 estaba a 10 metros del lugar donde los pobladores aledaños vierten aguas domésticas. El punto M6 estaba ubicado a 50 metros de cualquier población humana cercana y es el hábitat de peces y de aves. El punto M7 se encuentra cercana a la pista y donde los pobladores aledaños arrojan desperdicios. $\mathrm{Y}$ el punto M8 se encuentra a 10 metros de la playa. En los cuatro 
puntos sus conductividades fueron superiores a los $1400 \mathrm{uS} / \mathrm{cm}$. Pero sus niveles de salinidad fueron ínfimos por debajo de las 63ppt. Así como, su nivel de amoniaco total fue menor a lo permitido. Sin embargo, es resaltable el nivel alto de Nitratos en el punto M7 $\left(\mathrm{ECA}=13 \mathrm{mg} / \mathrm{L} \mathrm{NO}_{3}\right)$.

En la presente investigación se demostró que existe la presencia de xenobióticos contaminantes por actividad urbana y/o rural cercana principalmente con Nitrato sobre los principales humedales de la ciudad de Lima. Que afectan el crecimiento radicular de los meristemos de las liliáceas (Cebolla y ajo). Así mismo, se evidenció la afectación de la división celular pudiéndose afectar a las especies de flora y fauna propias de estos sistemas lacustres costeros.

\section{REFERENCIAS}

1. Ramsar (2018). ¿Qué son los humedales? Documento informativo No. 1. Recuperado de: https://www.ramsar.org/sites/default/files/documents/library/info2007sp-01.pdf

2. Clarkson, B.R., Sorrell; B. \& Reeves, P. (2004). Handbook for Monitoring Wetland Conditions. Ministry for the Environment Sustainable Management Fund Project. Recuperado de: https://www.landcareresearch.co.nz/publications/researchpubs/handbook_wetland_condition.p df

3. Decreto Supremo №55-2006-AG. Disponen la categorización de la Zona Reservada los Pantanos de Villa. (2006, setiembre 01). Diario el Peruano, 327170-327173.

4. SERNANP (2016). Recuperado de: http://www.sernanp.gob.pe/

5. Guillen Aguirre, Gisella. (2002). Diversidad protozoológica de los Pantanos de Villa, Chorrillos - Lima - Perú. Tesis de pregrado para optar el título de Biólogo. Facultad de Ciencias. Universidad Nacional Mayor de San Marcos.

6. León, B., Cano, A. \& Young, R. (1995). La flora vascular de los Pantanos de Villa, Lima, Perú: Adiciones y guía para las especies comunes. Publicaciones del Museo de Historia NaturalUNMSM. (B) 38:1-39.

7. Alvarez Begazo, Cristian (2007). Evaluación de la diversidad específica de las aves de los humedales de Ventanilla. Tesis de pregrado para optar el título de licenciado en ciencias biológicas de la Facultad de Ciencias de la Universidad Ricardo Palma.

8. Vizcardo, C. \& Gil-Kodaka, P. (2015). Estructura de las comunidades macrozoobentónicas de los humedales de Ventanilla, Callao, Perú. Anales Científicos, 76 (1): 1-11.

9. Tatum, E. (1947). Mutaciones químicamente inducidas y su rodamiento de la carcinogénesis. Anales de la Academia de Ciencias de Nueva York, 49: 87-97 
10. Kilbey, B. 1995. Charlotte Auerbach (1899-1994). Genetics. September 1, 141 (1): 1-5.

11. Madigan, M., Martinko, J., Stahl, D., Clark, \& Brock, D. Biology of Microorganisms. 13th Edition. 2010.

12. Wilhm, J. L. Biological Indicators of pollution. en B.A. Whitton, editor. River Ecology. Studies in ecology. Volumen 2. University of California Press. Berkeley, California. p.p. 375-402. (1975).

13. Saldaña, F. P., Sandoval, M. J. C., López, L. R. y Salcedo, S. E. (2001). Utilización de un índice de Diversidad para determinar la calidad del agua en sistemas loticos. Ingeniería Hidráulica en México, 16(2):57-66.

14. Vázquez G., Castro G., González, I., Pérez R. \& Castro T. 2006. Bioindicadores como herramientas para determinar la calidad del agua. Contactos, 60: 41-48.

15. Díaz Baez, M., Ronco, A. \& Pica Granados, Y. (2008). Ensayo de toxicidad aguda con bulbos de cebolla Allium cepa L. mediante la evaluación de la inhibición del crecimiento promedio de raíces. En: Ramirez Romero, P. y Mendoza Cantu, A. (comp). Ensayos toxicológicos para la evaluación de sustancias químicas en agua y suelo. La experiencia en México. p. 33-40. Secretaria de Medio Ambiente y Recursos Naturales. Instituto Nacional de Ecología.

16. Castillo Morales, Gabriela (ed.). Ensayos toxicológicos y métodos de evaluación de calidad de aguas. Estandarización, intercalibración, resultados y aplicaciones. México: IMTA, 2004.

17. Fiskesjö, G. (1993). The Allium Test in Wastewater Monitoring. Env. Toxicol. Wat. Qual., 8: 291298.

18. Fiskesjö, G. (1997). Allium Test for Screening Chemicals; Evaluation of Cytological Parameters", in: Plants for Environmental Studies, Wancheng, W.; J. W. Gorsuch, J. S. Hughes eds., CRC Press, Florida, pp. 308-329.

19. Decreto Supremo №15-2015-MINAM. Modifican los Estándares Nacionales de Calidad Ambiental para Agua y establecen disposiciones complementarias para su aplicación. (2015, diciembre 19). Diario el Peruano, 569076.

20. Decreto Supremo №004-2017-MINAM. Aprueban Estándares de Calidad Ambiental (ECA) para Agua y establecen Disposiciones Complementarias (2017, Junio 07). Diario el Peruano, p 10.

21. Brewster, J. (2001). Las cebollas y otros Alliums. Zaragoza, España: Editorial ACRIBIA, S.A.

22. Munns, R. y Tester, M. (2008). Mechanism of salinity tolerance. Annu. Rev. Plant Biol. 59, 65181.

Correo de contacto:

Email: carlosscottoespinoza@gmail.com 